\title{
Prevalence and distribution of colonic diverticula assessed with CT colonography (CTC)
}

\author{
Carlo Nicola De Cecco ${ }^{1,2} \cdot$ Maria Ciolina $^{1} \cdot$ Bruno Annibale $^{3} \cdot$ Marco Rengo $^{1} \cdot$ \\ Davide Bellini $^{1}$ - Giuseppe Muscogiuri ${ }^{1}$ - Antonello Maruotti ${ }^{4,5}$ - Luca Saba ${ }^{6}$. \\ Franco Iafrate $^{1}$ - Andrea Laghi ${ }^{1}$
}

Received: 28 December 2014 /Revised: 20 May 2015 / Accepted: 27 May 2015

(C) European Society of Radiology 2015

\begin{abstract}
Objectives This study aimed to evaluate the prevalence of colonic diverticula according to age, gender, distribution, disease extension and symptoms with CT colonography (CTC). Methods The study population included 1091 consecutive patients who underwent CTC. Patients with diverticula were retrospectively stratified according to age, gender, clinical symptoms and colonic segment involvement. Extension of colonic diverticula was evaluated using a three-point quantitative scale. Using this data, a multivariate regression analysis was applied to investigate the existence of any correlation among variables.

Results Colonic diverticula were observed in 561 patients (240 men, mean age $68 \pm 12$ years). Symptomatic uncomplicated diverticular disease (SUDD) was present in $47.4 \%$ of cases. In $25.6 \%$ of patients $\leq 40$ years, at least one
\end{abstract}

Andrea Laghi

andrea.laghi@uniroma1.it

1 Department of Radiological Sciences, Oncology and Pathology, University of Rome "Sapienza" - Polo Pontino, Via Franco Faggiana, 34, 04100 Latina, Italy

2 Department of Radiology \& Radiological Sciences, Medical University of South Carolina, 25 Courtenay Drive, Charleston, SC 29425, USA

3 Department of Digestive and Liver Disease, University of Rome "Sapienza" - Sant'Andrea Hospital, Via di Grottarossa 1035, Rome, Italy

4 Department of Public Institutions, Economy and Society, University "Roma Tre", Rome, Italy

5 Southampton Statistical Sciences Research Institute \& School of Mathematics, University of Southampton, Southampton, UK

6 Department of Radiology, Azienda Ospedaliera Universitaria di Cagliari, Cagliari, Italy diverticulum in the colon was observed. Prevalence of rightsided diverticula in patients $>60$ years was $14.2 \%$ in caecum and $18.5 \%$ in ascending colon. No significant difference was found between symptomatic and asymptomatic patients regarding diverticula prevalence and extension. No correlation was present between diverticula extension and symptoms.

Conclusion The incidence of colonic diverticula appears to be greater than expected. Right colon diverticula do not appear to be an uncommon finding, with their prevalence increasing with patient age. SUDD does not seem to be related to diverticula distribution and extension.

Key Points

- Incidence of colonic diverticula appears to be greater than expected.

- Right colon diverticula do not appear to be an uncommon finding.

- SUDD does not seem to be related to diverticula distribution and extension.

Keywords CT colonography · Diverticular disease · Diverticulosis $\cdot$ Colon $\cdot$ Abdominal pain

\section{Introduction}

Colonic diverticula are an acquired condition defined as the presence of small outpouchings of the colonic mucosa through the outer muscular layers at sites of vascular perforation [1]. This condition is defined as diverticulosis when asymptomatic and as diverticular disease when associated with clinical symptoms. Diverticular disease is further subclassified into symptomatic uncomplicated diverticular disease (SUDD) and symptomatic complicated diverticular disease, when perforation, fistula, obstruction and/or bleeding are present [2]. 
In industrialized Western countries, diverticulosis shows a high prevalence, affecting $5 \%$ of people in their fifth decade and approximately $50 \%$ of those in their ninth decade [3], with an estimated mortality rate of 2.5 per 100,000 per year. This represents the fifth most important gastrointestinal disease in terms of direct or indirect healthcare costs [4].

Studies on the prevalence of diverticulosis are mainly based on colonoscopy, necroscopy and barium enema [2]. In particular, before the advent of CT, which showed an excellent sensitivity in acute diverticulitis detection of 85-97 \% [5,6], barium enema was the most utilized imaging technique for demonstrating the presence of diverticula in symptomatic patients.

In recent years, CT colonography (CTC) has become a non-invasive alternative test for colorectal cancer screening, thanks to its excellent diagnostic accuracy $[7,8]$. Likewise, CTC can accurately detect the presence of diverticula and provides important clinical information, facilitating the selection of appropriate management [9]. Although CTC allows for accurate visualization of the whole colon and detection of the presence of diverticula, no systematic studies on disease prevalence and distribution have ever been performed using CTC.

Hence, the purpose of the current study was to evaluate the prevalence of diverticulosis and SUDD according to age, gender, colonic segments, disease extension and symptoms in a group of patients who underwent CTC.

\section{Materials and methods}

\section{Subjects}

The study population included 1091 consecutive Caucasian patients who underwent CTC examination between October 2007 and February 2012 for colon cancer screening and various clinical indications: positive faecal occult blood test, family history of colorectal cancer, atypical abdominal pain, incomplete colonoscopy, rectal bleeding, anaemia and weight loss. Patients who met any of the following criteria were not included in the study: (a) suspected or known inflammatory bowel disease; (b) colorectal cancer; (c) known colonic diverticula; (d) history of diverticulitis; (e) acute diverticulitis, defined as the presence of fever or elevated inflammatory markers (high white blood cell count, elevated C-reactive protein level, erythrocyte sedimentation rate increment); (f) acute abdominal pain.

\section{CTC protocol}

Protocol for CTC examination included a fibre-free diet 3 days before examination and bowel preparation. In detail, starting 3 days before the examination, patients were instructed to avoid intake of all fibre-rich food, including fruit, vegetables, whole-grain bread and whole-grain cereals. Bowel preparation was obtained with "fecal tagging" by administering an oral iodinated contrast agent to patients in a "1-day preparation". The iodinated contrast agents used were diatrizoate meglumine and diatrizoate sodium with an iodine concentration of $370 \mathrm{mg} / \mathrm{mL}$ (Gastrografin; Schering, Berlin, Germany) or Iopamidol with an iodine concentration of $300 \mathrm{mg} / \mathrm{mL}$ (Gastromiro, Bracco, Italy). Specifically, patients were asked to drink $170 \mathrm{~mL}$ of contrast agent diluted in a glass of water during a mild lunch $(85 \mathrm{~mL})$ and again at midafternoon $(85 \mathrm{~mL})$ followed by at least $1 \mathrm{~L}$ of water the day before the examination. In case of reported allergy to iodinated contrast media, patients were asked to consume cathartic drugs. In these particular cases, patients were asked to drink a solution consisting of 100 g PEG-4000 (ISOCOLAN, Bracco, Italy) and $2 \mathrm{~L}$ of water the evening before examination.

On the day of CTC examination, patients assumed a left lateral decubitus position so that a thin, lubricated, silicone Foley catheter could be gently inserted into the rectum. Colonic distention was obtained with room-air or with $\mathrm{CO}_{2}$ (PROTOCO2L, Bracco Diagnostics) according to patient tolerance (2.5 $\mathrm{L}$ of $\mathrm{CO}_{2}$ was the average amount). In case of inadequate colonic segmental distention a muscle relaxant, $20 \mathrm{mg}$ of butylscopolamine bromide (Buscopan ${ }^{\circledR}$ ), was intravenously or intramuscularly injected.

Supine and prone acquisitions were obtained with 64channel multi-detector CT system (Sensation Cardiac, Siemens), with $1.25 \mathrm{~mm}$ collimation, $\mathrm{kVp}$ of $120,50-75 \mathrm{mAs}$ and $1.5 \mathrm{~mm}$ reconstruction interval.

CTC images were retrospectively evaluated by two radiologists experienced in CTC interpretation (more than 5000 cases), using a primary 2D axial approach (primary window setting $1500,-250 \mathrm{HU}$ ) on a dedicated workstation (IM3D, Turin, Italy).

\section{Patient analysis}

Two abdominal radiologists with more than 5 years of experience recorded the number and location of diverticula for each patient. A three-point graded-scale scoring system was used to score the extension of colonic diverticula in each colonic segment: grade 1 (rare, less than six diverticula), grade 2 (multiple, 6-20) and grade 3 (severe, more than 20) (Fig. 1). Patients were considered affected by severe diverticulosis if at least one colonic segment was grade 3. Patients with diverticula were retrospectively stratified into seven groups according to age. Each group was further stratified according to gender, clinical symptoms and colonic segments involvement (caecum, ascending colon, transverse colon, descending colon and sigmoid colon). The rectum was not included in this analysis owing to the general absence of diverticula in this segment. 
Fig. 1 Diverticula grading scale. a grade 1 (rare, $<6$ diverticula); $\mathbf{b}$ grade 2 (multiple, $\geq 6-\leq 20$ ); c grade 3 (severe, $>20$ )

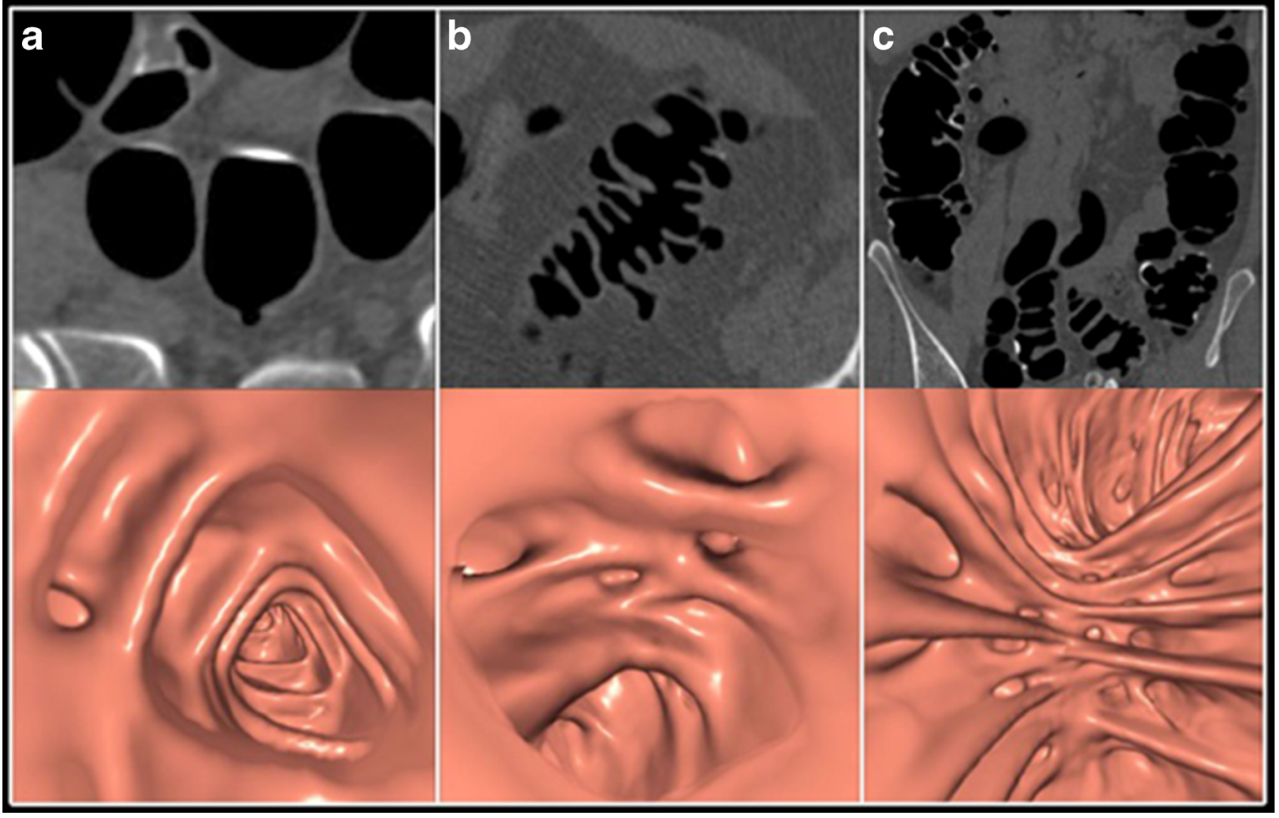

Diverticulosis was defined as the presence of colonic diverticula in absence of any clinical symptoms. Symptomatic uncomplicated diverticular disease (SUDD) was defined as the presence of colonic diverticula plus at least one point reported by the patient in the standardized questionnaire used to record patients' history and symptoms: (1) periodic abdominal pain/discomfort; (2) history of diarrhoea/constipation; (3) alternating bowel habits; (4) abdominal tenderness/bloating; (5) history of rectal bleeding. Since a validated specific questionnaire for SUDD is lacking, symptoms were evaluated by means of a validated questionnaire for dyspeptic and abdominal symptoms according to Rome III criteria [10,11].

CT signs of acute uncomplicated diverticulitis were considered as mural thickening, increased fatty attenuation adjacent to the afflicted segment, free peritoneal fluid and enlarged lymph nodes [12]. Mural abscesses or extramural abscesses, fistulas and colonic perforation were reported in cases of acute complicated diverticulitis. CT signs of chronic uncomplicated diverticulitis were considered severe mural thickening with reduced colonic distention and pericolic fat stranding [13].

\section{Statistical analysis}

Descriptive statistics are provided as mean \pm standard deviation (SD) for continuous variables and absolute and relative frequencies for categorical variables. Normal data distribution was assessed with a Kolmogorov-Smirnov test. Student's two-tailed $t$ test for paired samples was performed to evaluate differences in diverticula prevalence and distribution related to patient gender, symptoms and CT signs. In order to correlate the diverticular prevalence and severity with the symptoms and CT signs, a multivariate regression analysis was performed. Statistical analysis was carried out using SPSS (version 21.0; SPSS, Chicago, IL, USA), and a $p$ values of less than 0.05 were considered statistically significant.

\section{Results}

\section{Diverticular distribution and severity}

Of a total population of 1091 Caucasian patients, diverticula were observed in 561 patients with an overall prevalence of $51.6 \%, 240(42.8 \%)$ men, and a mean age of $68 \pm 12$ years (Table 1).

Diverticula were distributed as follows: caecum, 125 (9.6\%) segments; ascending colon, 172 (13.1\%); transverse colon, 167 (12.7\%); descending colon, 355 (27.1\%); sigmoid colon, 492 (37.5\%) (Fig. 2) for a total of 1311 segments involved. Diverticula were graded as rare in 138 patients (24.6\%), multiple in 193 (34.4\%) and severe in $230(41.0 \%)$.

No significant difference in diverticula prevalence and distribution was observed related to patient gender $(p>0.05)$.

The overall prevalence of diffuse diverticulosis according to patients' age is higher in left-sided colon than right-sided colon. In particular, in the sigmoid colon, the overall prevalence was $51.2 \%$ in the 6 th decade of life, $48.4 \%$ in the 7 th decade and $67.1 \%$ in the 8 th decade, while it ranged between $23.3 \%$ and $36.4 \%$ during the 4 th and 5 th decades. Considering the right colon, the highest overall prevalence was found in the 6th and 7th decades, occurring in 17.3-18.7\% of patients in the ascending colon and $14.2-12.7 \%$ in the caecum.

Disease extension increased with age in all colonic segments, especially in the sigmoid colon where grade 3 ranged from $2.5 \%$ for patients under 40 years of age to $28.6 \%$ for those older than 70 years (Table 1 ). 


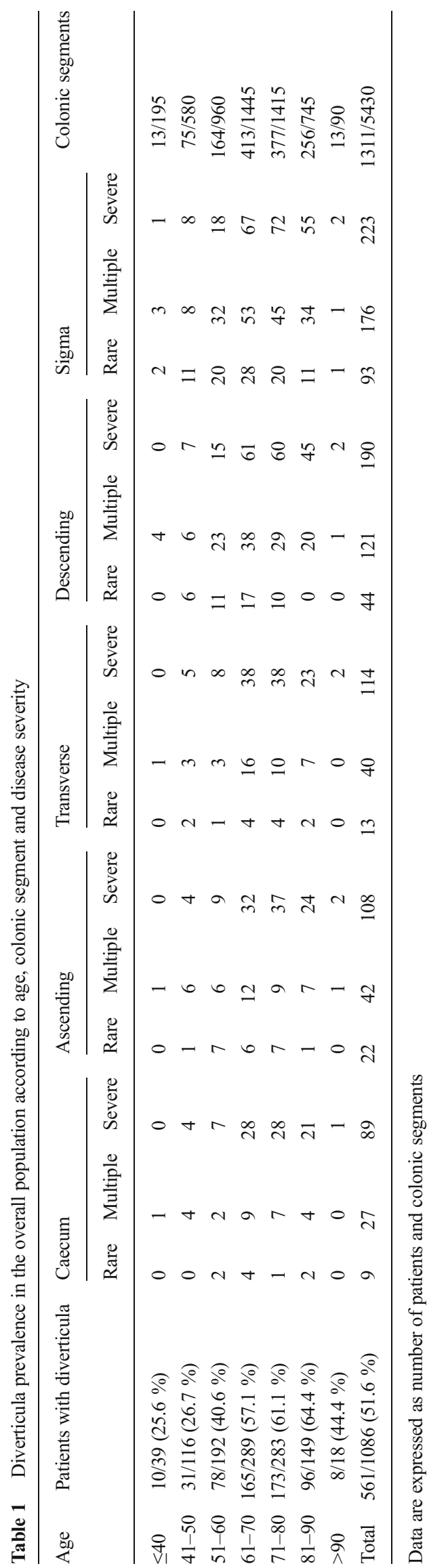

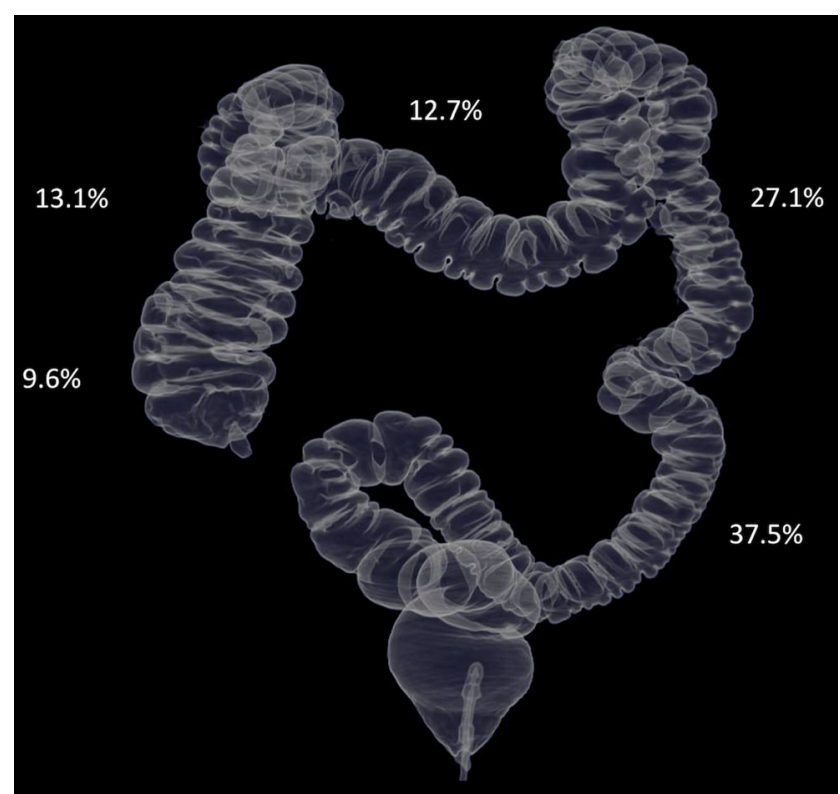

Fig. 2 Diverticula prevalence in the overall population

In $25.6 \%$ of patients under 40 years of age at least one diverticulum per patient was observed considering all the colonic segments, and in $15.4 \%$ of patients diverticula were located in the sigmoid colon.

\section{Diverticula and symptoms}

SUDD was present in 266 (47.4\%) patients with diverticula. No correlation was found between distribution or extension of diverticula and symptoms $(r=0.3 ; p>0.05)$. No significant difference was found between symptomatic and asymptomatic patients regarding diverticula prevalence and extension $(p>0.05)$.

The highest prevalence of SUDD occurred in the 6th and 7th decades in the left sided colon (41.2-39.9\% in sigmoid colon and $33.9-30.0 \%$ in descending colon). In the same decades, the prevalence of SUDD in the right colon was $15.7-17.3 \%$ in the ascending segment and $13.3-10.4 \%$ in the caecum (Table 2).

In asymptomatic patients, the highest prevalence of severe diverticulosis was found in the left-sided colon in the 6th and 7 th decades. In particular, prevalence was $17.5-20.8 \%$ in the sigmoid colon, $15.7-16.2 \%$ in the descending colon, 9.7$8.7 \%$ in the transverse colon, $6.7-9.8 \%$ in the ascending colon and $6.7-7.5 \%$ in the caecum (Table 3).

Diverticulitis CT signs were found with a prevalence of $3.7 \%(21 / 561)$ while prevalence of CT signs of chronic diverticulitis was $4.1 \%(23 / 561)$. No CT signs of acute complication (stenosis, abscess) were observed in any patients. All patients with signs of acute diverticulitis were symptomatic; in contrast only $47.8 \%(11 / 23)$ of patients with chronic uncomplicated diverticulitis were symptomatic. 

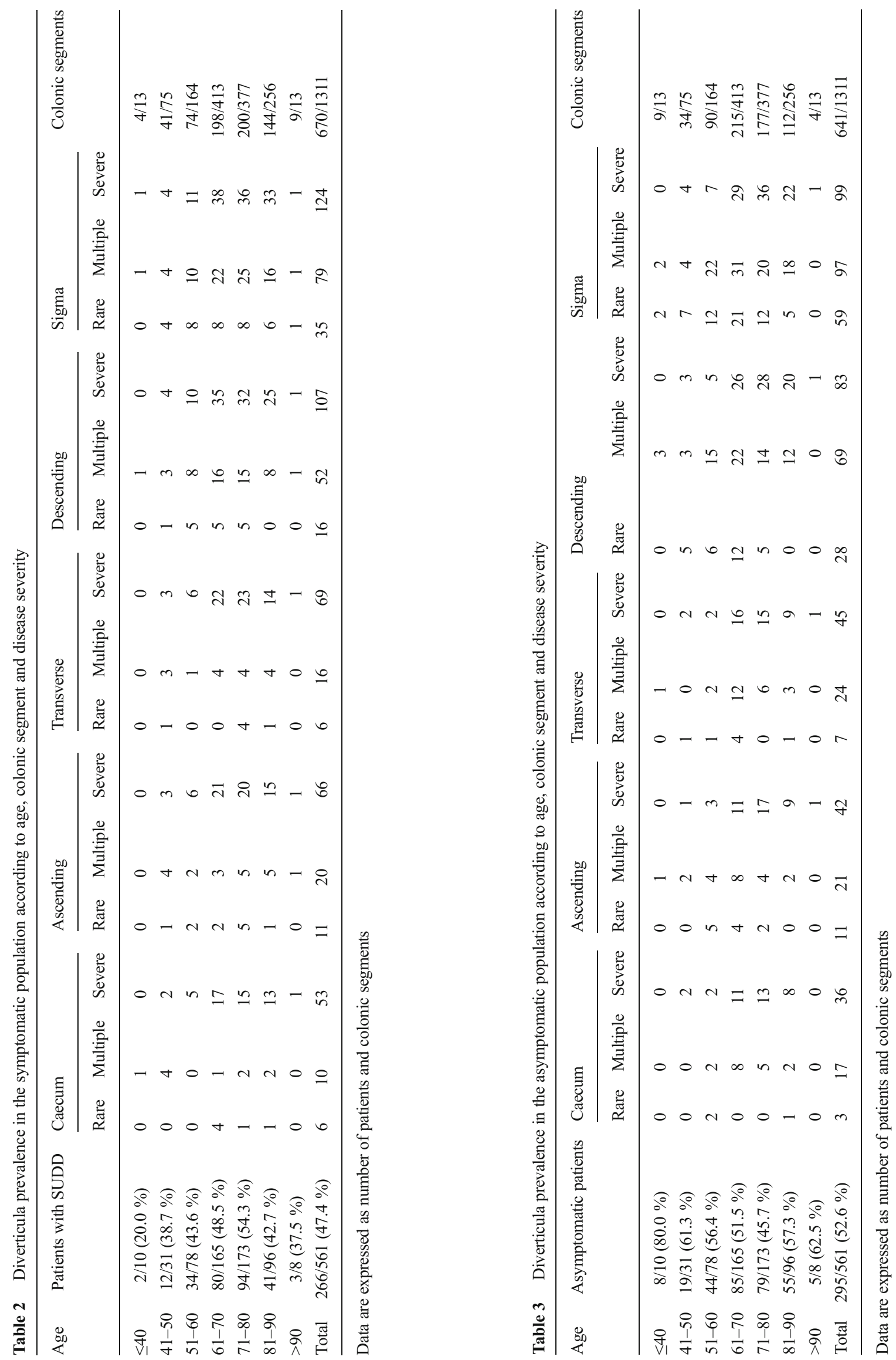


\section{Discussion}

CTC has become the non-invasive imaging technique of choice for assessment of the colon owing to its ability to accurately depict the colonic wall. This represents the first study evaluating prevalence and distribution of colonic diverticular and associated symptoms on a large patient population studied with CTC. Other studies have previously reported initial data on detection of colonic diverticula using CTC $[14,15]$, but those studies were performed on a limited number of patients and mainly focused on the sigmoid colon; moreover, they were neither specifically aimed at assessing diverticula distribution and extension according to age and gender nor their association with clinical symptoms.

We found that the overall prevalence of diverticula $(47.3 \%)$ was similar compared with a recent European study [16] over a population of 1000 patients that underwent barium enema. This value is significantly higher than previously reported (15-35\%) [17-19], supporting the idea that diverticula prevalence in Western countries is increasing, likely attributed to population aging and dietary changes [20]. Likewise, the mean age of patients with diverticula in our study was $68 \pm$ 12 years, demonstrating a steady increment of the disease prevalence with increased age. Moreover, age was the strongest predictor of diverticula, as previously reported [19,21].

Although colonic diverticula are an uncommon pathological condition for young patients, in our study it was observed that $25.6 \%$ of subjects aged 40 years or less presented at least one diverticulum in the colon. This data appears in line with previous studies performed in young patients with barium enema, standard abdominal CT or surgical intervention, where the prevalence of diverticula in patients younger than 40 years old was 5-29\% [22-27].

Regarding the right-sided diverticular disease, our results support the hypothesis that in the Western population it is not an uncommon finding. In fact, we found a prevalence of rightsided diverticula in patients older than 60 years ranging from $14.2 \%$ of the caecum to the $18.5 \%$ of the ascending colon, in line with the prevalence of $10-17 \%$ previously reported in a Caucasian population [18-20]. Recently, a higher prevalence $(28 \%)$ of right-sided diverticulosis in Western populations was reported in a barium study [17]. However, in the barium studies the pan-diverticular disease was responsible for a significant relative contribution to the total of right-sided diverticula. In our study, the disease prevalence in the right colon increased with age, supporting also the idea that, similar to left-sided diverticula, the majority of right-sided diverticula are acquired, rather than congenital [28,29].

From our results, gender does not seem to be related to diverticula prevalence and distribution. These data are consistent with previous studies demonstrating no difference in gender distribution between age classes in patients with diverticular disease [30,31]. A recent study reported instead that with increasing age, the proportion of women with SUDD significantly increases compared to men, while younger age groups with diverticular disease are more likely to consist of men [11]. This observation could be the result of better healthcare seeking rates in younger groups and an increased prevalence of women in older groups.

According to the clinical presentation, SUDD was present in $47.4 \%$ of cases. However, no correlation was found between extension of diverticulosis and symptoms. Our finding is consistent with the previously reported observation that a clear correlation between episodes of unspecific abdominal pain and the presence of diverticulosis is hard to find [32,33], since these symptoms are present in other pathological conditions, such as irritable bowel syndrome (IBS) or inflammatory bowel disease (IBD), and thus they may be indistinguishable from those of SUDD, representing an important source of overlap [34]. In this clinical setting, CTC could represent an effective non-invasive imaging technique to rule out with high accuracy the presence of diverticular disease, and attribute the patient symptoms to other causes of abdominal pain.

Our findings should be analysed in the light of the study limitations. In particular, patients' symptoms were collected through an auto-assessment questionnaire, which is prone to bias, related to subjective symptoms reporting. In addition, a small number of patients younger than 40 years were included in this retrospective analysis since the main indication for CTC was represented by colorectal cancer screening which starts usually at age 50 in individuals at average risk of colorectal cancer. This could represent a bias for the effective assessment of juvenile diverticular disease prevalence. Finally, the population studied was mainly composed of patients who underwent CTC for colorectal cancer screening, and thus could not be representative of the general population. In any case, we should be aware that previous studies performed both with colonoscopy and barium enema were likely to be affected by the same bias.

In conclusion, CTC depicted a different colonic diverticula scenario in comparison to previous autoptic and barium enema studies. The diverticula incidence appears to be higher than expected without significant differences according to gender. In the Western population, right colon diverticula do not appear to be an uncommon finding, with their prevalence increasing with patient age. SUDD seems to be unrelated to diverticula distribution and extension.

Acknowledgements The scientific guarantor of this publication is Andrea Laghi. The authors of this manuscript declare no relationships with any companies whose products or services may be related to the subject matter of the article. The authors state that this work has not received any funding. Antonello Maruotti from University "Roma Tre" kindly provided statistical advice for this manuscript. Institutional review board approval was obtained. Written informed consent was obtained from all subjects (patients) in this study. All study subjects or cohorts have not been previously reported. Methodology: retrospective, observational, performed at one institution. 


\section{References}

1. Stollman N, Raskin JB (2004) Diverticular disease of the colon. Lancet 363:631-639

2. Kohler L, Sauerland S, Neugebauer E (1999) Diagnosis and treatment of diverticular disease: results of a consensus development conference. The scientific committee of the european association for endoscopic surgery. Surg Endosc 13:430-436

3. Petruzziello L, Iacopini F, Bulajic M et al (2006) Review article: uncomplicated diverticular disease of the colon. Aliment Pharmacol Ther 23:1379-1391

4. Sandler RS, Everhart JE, Donowitz M et al (2002) The burden of selected digestive diseases in the United States. Gastroenterology 122:1500-1511

5. Pradel JA, Adell JF, Tauorel P et al (1997) Acute colonic diverticulitis: prospective comparative evaluation with $\mathrm{CT}$ and US. Radiology 205:503-512

6. Rao PM, Rhea JT, Novelline RA et al (1998) Helical CT with only colonic contrast medium for diagnosing diverticulitis. Am J Roentgenol 170:1445-1449

7. Laghi A, Rengo M, Graser A, Iafrate F (2013) Current status on performance of CT colonography and clinical indications. Eur J Radiol 82:1192-1200

8. Spada C, Stoker J, Alarcon O et al (2015) Clinical indications for computed tomographic colonography: european society of gastrointestinal endoscopy (ESGE) and european society of gastrointestinal and abdominal radiology (ESGAR) guideline. Eur Radiol 25: 331-345

9. Halligan S, Saunders B (2002) Imaging diverticular disease. Best Pract Res Clin Gastroenterol 16:595-610

10. Drossman DA, Corazziari E, Delvaux M et al (2006) Rome III, 3rd edn, The functional gastrointestinal disorders. Degnon Associates, McLean, pp pp 1-pp 29

11. Annibale B, Lahner E, Maconi G, Usai P, Marchi S, Bassotti G et al (2012) Clinical features of symptomatic uncomplicated diverticular disease: a multicenter Italian survey. Int J Colorectal Dis 27:11511159

12. Buckley O, Geogheganb T, O'Riordaina DS, Lyburnc ID, Torreggiani WC (2004) Computed tomography in the imaging of colonic diverticulitis. Clinic Radiol 59:977-983

13. Flor N, Rigamonti P, Pisani Ceretti A, Romagnoli S, Balestra F, Sardanelli F et al (2013) Diverticular disease severity score based on CT colonography. Eur Radiol 23:2723-2729

14. Gollub MJ, Jhaveri S, Schwartz E, Felderman H, Cooper C, Markowitz AJ et al (2005) CT colonography features of sigmoid diverticular disease. Clin Imaging 29:200-206

15. Ghuman M, Bates N, Moore H (2012) Computed tomographic colonography (CTC): a retrospective analysis of a single site experience and a review of the literature on the status of CTC. N Z Med J 125:60-67

16. Golder M, Ster IC, Babu P et al (2011) Demographic determinants of risk, colon distribution and density scores of diverticular disease. World J Gastroenterol 17:1009-1017

17. Blachut K, Paradowski L, Garcarek J (2004) Prevalence and distribution of the colonic diverticulosis. Review of 417 cases from Lower Silesia in Poland. Rom J Gastroenterol 13:281-285

18. Manousos ON, Truelove SC, Lumsden K (1967) Transit times of food in patients with diverticulosis or irritable colon syndrome and normal subjects. Br Med J 3:760-762

19. Koehler R (1963) The incidence of colonic diverticulosis in Finland and Sweden. Acta Chir Scand 126:148-155

20. Roberts P, Abel M, Rosen L et al (1995) Practice parameters for sigmoid diverticulitis. The standards task force american society of colon and rectal surgeons. Dis Colon Rectum 38:125-132

21. Debray C, Hardouin JP, Besancon F, Raimbault J (1961) Incidence of colic diverticulosis according to age. Statistical study from 500 barium enemas. Sem Hop 37:1743-1745

22. Eusebio EB, Eisenberg MM (1973) Natural history of diverticular disease of the colon in young patients. Am J Surg 125:308-311

23. Ouriel K, Schwartz SI (1983) Diverticular disease in the young patient. Surg Gynecol Obstet 156:1-5

24. Simonowitz D, Paloyan D (1977) Diverticular disease of the colon in patients under 40 years of age. Am J Gastroenterol 67:69-72

25. Chodak GW, Rangel DM, Passaro E Jr (1981) Colonic diverticulitis in patients under age 40: need for earlier diagnosis. Am J Surg 141: 699-702

26. Faria GR, Almeida AB, Moreira $\mathrm{H}$ et al (2011) Acute diverticulitis in younger patients: any rationale for a different approach? World $\mathrm{J}$ Gastroenterol 17:207-212

27. Markham NI, Li AK (1992) Diverticulitis of the right colon - experience from Hong Kong. Gut 33:547-549

28. Hughes LE (1969) Postmortem survey of diverticular disease of the colon. Gut 10:336-351

29. Rodky GV, Welch CE (1984) Changing patterns in the surgical treatment of diverticular disease. Ann Surg 200:466-478

30. Jung H-K, Choung RS, Locke GR 3rd, Schleck CD, Zinsmeister AR, Talley NJ (2010) Diarrhea-predominant irritable bowel syndrome is associated with diverticular disease: a population-based study. Am J Gastroenterol 105:652-661

31. Parra-Blanco A (2010) Colonic diverticular disease: pathophysiology and clinical picture. Digestion 73(Suppl 1):47-57

32. Lee KM, Paik CN, Chung WC et al (2010) Clinical significance of colonic diverticulosis associated with bowel symptoms and colon polyp. J Korean Med Sci 25:1323-1329

33. Salemi TA, Molloy RG, O’Dwyer PJ (2007) Prospective, five-year follow-up study of patients with symptomatic uncomplicated diverticular disease. Dis Colon Rectum 50:1460-1464 